\title{
Internationale Produktionsstrategien bei Automobilzulieferern - Erfahrungen und Muster
}

\author{
Steffen Kinkel \\ Christoph Zanker
}

\begin{abstract}
Entscheidungen über den Aufbau internationaler Produktionsstandorte sind angesichts der Globalisierung vieler produzierender Unternehmen unumgänglich geworden. Automobilzulieferer sind besonders häufig betroffen. Verschiedene Beweggründe, wie Kostenreduktion, Markterschließung oder Marktnähe zum Kunden, stellen zunehmend auch kleine und mittlere Zulieferer vor derartige Entscheidungen - und gerade für sie können unzureichend fundierte Kalküle existenzielle Folgen haben. Der Beitrag zeigt Erfolgsmuster und zentrale Entscheidungsfaktoren internationaler Produktionsstrategien bei Automobilzulieferern auf und stellt auf dieser Grundlage neue Ansätze zur strategisch fundierten und zukunftsorientierten Standortbewertung vor.
\end{abstract}

\section{Einleitung}

Standortentscheidungen im internationalen Kontext sind unternehmerische Grundsatzentscheidungen, die wegen ihres langfristigen Einflusses auf die Wettbewerbsfähigkeit der Unternehmen und auf das Arbeitsplatzangebot in Deutschland von höchster strategischer Bedeutung sind. Entscheidungen zur Ansiedlung von Produktionsstandorten sind dabei aufgrund ihrer unmittelbaren und mittelbaren Beschäftigungs- und Ausstrahleffekte von besonderem Interesse (Dreher 2004). Heute ist der Aufbau einer Auslandsproduktion nicht mehr nur ein Aktionsfeld für die großen, multinational agierenden ,global player", sondern zunehmend auch für kleine und mittlere Unternehmen (Bassen et al. 2001; DIHK 2003). Die Erweiterung der EU um zehn neue Mitgliedsländer hat dieser Entwicklung eine zusätzliche Dynamik verliehen.

Dabei ist unstrittig, dass die deutsche Industrie bislang von ihrer internationalen Ausrichtung profitiert hat. Internationale Wertschöpfungsstrategien zur Marktbearbeitung vor Ort oder zur Sicherung einer den Markterfordernissen angemessenen Mischkalkulation haben auch bei deutschen Betriebsstandorten zu erhöhter Wettbewerbsfähigkeit und Wachstum beigetragen. Neuere Befunde werfen aber teilweise auch kritische Schlaglichter auf einige Formen der Auslandsproduktion, insbesondere auf kostengetriebene Verlagerungs- und Outsourcing-Strategien. Empirische Erkenntnisse zeigen, dass diese im Gegensatz zu markt- und kundenorientierter Aus- landsproduktion mit negativen Konsequenzen für den heimischen Arbeitsmarkt behaftet sind (IKB/KfW 2004; Kinkel et al. 2004). Zudem gibt es Anzeichen, dass kostenorientierte Verlagerungen häufiger als markt- und kundenorientierte scheitern und zu Rückverlagerungen führen (Kinkel 2004). Gerade stark kostengetriebene Standortentscheidungen scheinen demnach nicht immer alle relevanten Gesichtspunkte zu berücksichtigen und suboptimal getroffen zu werden (PA Consulting 2004; Abele et al. 2006).

Besonders dynamisch ist das Verlagerungs- und Rückverlagerungsverhalten bei Betrieben der deutschen Automobilzulieferindustrie (Kinkel et al. 2004; Lay et al. 2003). Automobilzulieferer nehmen für die deutsche Volkswirtschaft und das Arbeitsplatzangebot eine Schlüsselstellung ein. Auf Basis der Umfrage „Innovationen in der Produktion“ des Fraunhofer Instituts für System- und Innovationsforschung (ISI) und der Input-Output-Tabellen des Statistischen Bundesamtes für das Jahr 2000 lässt sich zeigen, dass im deutschen Verarbeitenden Gewerbe über alle Vorleistungsstufen hinweg etwa 990.000 Beschäftigte in der Automobilzulieferung tätig sind (Kinkel/Zanker 2006). Dieser Wert, der die reale Bedeutung der Automobilzulieferer belastbar widerspiegelt, liegt um den Faktor drei höher als die bislang verfügbaren statistischen Zahlen ${ }^{1}$ und sollte bei künftigen Analysen des Automobilzuliefersektors berücksichtigt werden.

Vor diesem Hintergrund wird im Folgenden analysiert, ${ }^{2}$ welche typischen internationalen Produktions- und Standortstrategien deutsche Automobilzulieferer verfolgen, welche zentralen Entscheidungsfaktoren sie dazu heranziehen und wie
Muster besonders erfolgversprechender Strategien aussehen. Zugleich wird herausgearbeitet, welche Defizite der Prozess der Standortentscheidungsfindung, der in den betrachteten Unternehmen nicht immer vollständig rational abläuft, aufweist und welche kritischen Faktoren häufig zu wenig beachtet oder falsch eingeschätzt werden. Auf diesen Ergebnissen aufbauend werden neue Ansätze zur strategisch fundierten und zukunftsorientierten Standortbewertung bei Automobilzulieferfirmen skizziert, die methodisch an den identifizierten Schwächen und Defiziten herkömmlicher

\footnotetext{
Diese umfassen nach der Systematik der Wirtschaftszweige in der Europäischen Gemeinschaft (NACE) die Unterklasse 34.3: „Hersteller von Teilen und Zubehör für Kraftwagen und Kraftwagenmotoren".

2 Die dargestellten Ergebnisse sind Teil des von der Hans-Böckler-Stiftung geförderten Forschungsvorhabens "Neue Methoden für strategisch fundierte Standortentscheidungen bei Automobilzulieferern".
}

Steffen Kinkel, Dr. rer. pol., Leiter der Abteilung Industrie- und Serviceinnovationen am Fraunhofer Institut für System- und Innovationsforschung (ISI). Arbeitsschwerpunkte: Internationale Produktion, Standortbewertung, innovative Geschäfts- und Organisationsmodelle, Evaluation, Foresight. e-mail: steffen.kinkel@isi.fraunhofer.de Christoph Zanker, Dipl. Kfm., Wissenschaftlicher Mitarbeiter in der Abteilung Industrieund Serviceinnovation am ISI.

Arbeitsschwerpunkte: Integrierte Modernisierungskonzepte, strategisches Controlling Standortentscheidungen.

e-mail: christoph.zanker@isi.fraunhofer.de 
Tabelle 1: Kenngrößen der drei Betriebstypen unterschiedlicher Standortstrategien

\begin{tabular}{|c|c|c|c|c|}
\hline Kenngrößen & $\begin{array}{c}\text { Sample- } \\
\text { Durchschnittswerte } \\
\text { Automobil- } \\
\text { zulieferer }\end{array}$ & $\begin{array}{c}\text { Typ 1: } \\
\text { Home based player } \\
\text { (keine Verlagerung) }\end{array}$ & $\begin{array}{c}\text { Typ 2: } \\
\text { Kostenorientierter } \\
\text { Auslandsproduzent }\end{array}$ & $\begin{array}{c}\text { Typ 3: } \\
\text { Markt- oder } \\
\text { kundenorientierter } \\
\text { Auslandsproduzent }\end{array}$ \\
\hline $\begin{array}{l}\text { Beschäftigungsentwicklung (-wachstum) } \\
\text { zwischen } 2000 \text { und } 2002 \text { - in \% pro Jahr - }\end{array}$ & 4,1 & 5,1 & $-1,9 * *$ & 3,8 \\
\hline $\begin{array}{l}\text { Wertschöpfungstiefe } \\
\text { ((Umsatz - Vorleistungen) / Umsatz) - in \% - }\end{array}$ & 60,9 & $64,4 * * *$ & 51,6 & 51,4 \\
\hline Anteil der Personalkosten - in \% - & 34,8 & 36,1 & 34,0 & $30,1^{*}$ \\
\hline Gemeinkostenanteil (Median) & 20,0 & 20,0 & 30,0 & 21,5 \\
\hline Abschreibungsquote - in \% - & 6,8 & $7,6 * *$ & 4,5 & 5,0 \\
\hline Anteil der FuE-Ausgaben - in \% - & 5,1 & 5,0 & $2,4^{* *}$ & 7,0 \\
\hline Umsatzrendite - in \% - & 5,6 & 5,9 & 3,8 & 4,6 \\
\hline
\end{tabular}

Standortentscheidungsprozesse ansetzen. Die Methoden sollen sowohl die Unternehmensführung wie auch Betriebsräte befähigen, Standortentscheidungen zu treffen bzw. nachvollziehen zu können, die mittel- bis langfristig tragfähige Lösungen versprechen. Gerade Betriebsräten und anderen Trägern der Mitbestimmung fehlt vielfach das methodische Rüstzeug und Wissen, um vorliegende Standortvergleichsrechnungen nachvollziehen und hinsichtlich ihrer inhärenten Schwächen einschätzen zu können.

\section{Drei Betriebstypen internationaler Produktionsstrategien}

\subsection{QUANTITATIVE BEFUNDE}

Vorliegende Studien und quantitative Ergebnisse (Abele et al. 2006; IKB/KfW 2004; Kinkel et al. 2004; Kinkel 2004; Lay et al. 2003) betonen die Notwendigkeit, zur Identifikation der maßgeblichen Standortfaktoren und Erfolgsmuster verschiedene Typen von Unternehmen zu betrachten, die sich hinsichtlich ihrer treibenden Motive bei der Standortwahl unterscheiden. Die dazu durchgeführten quantitativen Analysen haben gezeigt, dass sich Automobilzulieferer anhand ihrer Auslandsproduktionsaktivitäten und der dahinter stehenden Motive in drei Betriebstypen internationaler Produktionsstrategien einordnen lassen (Kinkel/Zanker 2006):

(1) Der Typ des Home based players siedelt seine Produktionskapazitäten weiterhin vorzugsweise oder ausschließlich am deutschen Standort an. Er bezieht seine Vorleis- tungen vorrangig von Zulieferern aus dem (erweiterten) regionalen Umfeld, zu denen oftmals langjährig eingespielte partnerschaftliche Beziehungen bestehen.

(2) Der Typ des kostenorientierten Auslandsproduzenten hat in den letzten Jahren verstärkt Investitionen in Niedriglohnländern zum Aufbau von Produktionskapazitäten getätigt. Er verfolgt primär die Intention, Faktorkosten und insbesondere Personalkosten durch zusätzliche Kapazitäten in Niedriglohnstandorte oder dorthin verlagerte Produktionsstätten zu reduzieren, um über die Möglichkeit verbesserter Mischkalkulationen seine preisliche Wettbewerbsfähigkeit zu optimieren.

(3) Der Typ des markt- und kundenorientierten Auslandsproduzenten baut Produktionswerke im Ausland vorrangig zur Markterschließung und Verbesserung der Kundennähe, oftmals auf konkrete Kundenanfrage hin, auf. Sein wichtigstes Ziel ist es, durch die Errichtung ausländischer Produktionsstätten entweder Aufträge von neuen ausländischen Kunden oder neue Aufträge von bestehenden Kunden, die ihrerseits Produktions- und Montagewerke im Ausland aufgebaut haben, zu erhalten bzw. neu zu erschließen.

$\mathrm{Zu}$ diesen drei Betriebstypen wurden vergleichende quantitative Analysen auf Basis der Daten der ISI-Umfrage Innovationen in der Produktion 2003 durchgeführt, die für den jeweiligen Typ durchaus repräsentativen Charakter haben. ${ }^{3}$ In der Datenbasis ist der Typ des Home based players dadurch abgegrenzt, dass er im Betrachtungszeitraum 2001 bis 2003 keine Produktionskapazitäten an ausländische Standorte verlagert hat. Der Typ des kostenorientierten Auslandsproduzenten hat im Zeitraum
2001 bis 2003 Produktionsverlagerungen ins Ausland durchgeführt, wobei die Reduktion der Faktorkosten ein entscheidendes Motiv war. Markt- und kundenorientierte Motive hatten dagegen keine Bedeutung. Der dritte Betriebstyp des marktund kundenorientierten Auslandsproduzenten hat zwischen 2001 und 2003 Produktionsverlagerungen ins Ausland vorgenommen, bei denen Markterschließung und Kundennähe mit von entscheidender Bedeutung waren. In der Stichprobe entfallen auf den ersten Betriebstyp $73 \%$, auf den zweiten $10 \%$ und auf den dritten etwa $17 \%$ der befragten deutschen Automobilzulieferer.

\section{BESCHÄFTIGUNGSENTWICKLUNG}

Insgesamt zeigen sich zwischen den drei Automobilzulieferertypen unterschiedlicher Standortstrategien teilweise signifikante Unterschiede bei der Ausprägung einiger Kenngrößen (Tabelle 1). So weisen kostenorientierte Auslandsproduzenten (Typ 2) als einziger der drei Typen mit im Mittel nahezu -2 \% in den Jahren 2000 bis 2002 eine negative Beschäftigungsentwicklung auf. Home based playern (Typ 1) wie

3 Für die Erhebungsrunde 2003 wurden 13.259 Betriebe aus Kernbereichen des deutschen Verarbeitenden Gewerbes (Metall- und Elektro-, Chemische und Kunststoffverarbeitende Industrie) angeschrieben, von denen 1.450 einen verwertbaren Fragebogen zurückgesandt haben (Rücklaufquote $11 \%)$. Wie Vergleiche der Stichprobe mit der Grundgesamtheit zeigen, weicht die Datenbasis der Umfrage hinsichtlich ihrer Branchenverteilung nur unwesentlich von der Grundgesamtheit $a b$ und repräsentiert neben großen Betrieben auch kleine und mittlere Unternehmen (KMU) sehr gut. Von den 1.450 Betrieben lassen sich 192 hinsichtlich der Frage, welche Branche sie vorrangig beliefern, als Automobilzulieferer einordnen. 
auch markt- und kundenorientierten Auslandsproduzenten (Typ 3) gelang es dagegen, im betrachteten Zeitraum auch am deutschen Betriebsstandort weiterhin Beschäftigung um etwa 4 bis $5 \%$ auszubauen. Damit bestätigen sich auch für den Bereich der Automobilzulieferer vorliegende Befunde für das Verarbeitende Gewerbe, wonach Produktionsverlagerungen aus Kostengründen tendenziell mit negativen Beschäftigungseffekten für den deutschen Standort einhergehen, während marktund kundenorientierte Auslandsproduktion zu Beschäftigungssicherung bzw. -wachstum an den inländischen Produktionsstandorten beiträgt (Kinkel et al. 2004).

\section{WERTSCHÖPFUNGSTIEFE}

Auch bei der Analyse der Wertschöpfungstiefe, errechnet als Anteil der internen Wertschöpfung (Umsatz minus Vorleistung) in Bezug zum Umsatz, zeigen sich deutliche Unterschiede. Der Typ des Home based players realisiert mit im Mittel etwa $64 \%$ eine deutlich höhere Eigenleistungstiefe als kostenorientierte Auslandsproduzenten bzw. markt- und kundenorientierte Auslandsproduzenten mit jeweils etwa $51 \%$. Gerade Betriebe, die bewusst stark auf ihre Einbettung in regionale Strukturen und Netzwerke setzen, scheinen also erfolgreich zu sein mit der Strategie, mehr als andere Betriebe „inhouse“ zu machen, und sich damit auch über die Tiefe der Eigenleistung im Wettbewerb behaupten zu können. Dagegen scheinen sowohl kostenorientierte Auslandsproduzenten als auch markt- und kundenorientierte Auslandsproduzenten stärker darauf zu setzen, über gezielte Outsourcing-Strategien geringere Wertschöpfungstiefen zu realisieren.

\section{PERSONAL- UND} GEMEINKOSTENANTEIL

Beim Vergleich des Personalkostenanteils zeigt sich, dass nur die markt- und kundenorientierten Auslandsproduzenten mit einem Personalkostenanteil von im Mittel etwa $30 \%$ eine leicht geringere Personalintensität ihrer inländischen Wertschöpfungsprozesse als die beiden anderen Betriebstypen aufweisen. Betriebe des Typs Home based player tolerieren dagegen bewusst die höheren Personalkosten am deutschen Standort zugunsten einer hohen Qualifikation, Flexibilität und Innova- tionsorientierung ihrer Belegschaft. Kostenorientierte Auslandsproduzenten nehmen wiederum den im Mittel höheren Personalkostenanteil zum Anlass, aktiv nach Möglichkeiten zur Reduktion der Personalkosten durch Auslandsverlagerungen im Produktionsbereich zu suchen.

Auch der jeweils ausgewiesene Gemeinkostenanteil, das heißt: der Anteil an den Selbstkosten, der für indirekte, nicht direkt verrechenbare Tätigkeiten anfällt, ist über die betrachteten Betriebstypen unterschiedlich. Demnach weisen kostenorientierte Auslandsproduzenten (Typ 2) mit einem Anteil aller Gemeinkosten an den Selbstkosten von insgesamt etwa $30 \%$ hier einen höheren Wert auf als die Home based player bzw. die markt- und kundenorientierten Auslandsproduzenten, die jeweils in der Lage sind, einen Gemeinkostenanteil von etwa $20 \%$ zu verwirklichen. Dies deutet - wenn auch schwach - darauf hin, dass bei kostenorientierten Auslandsproduzenten die aus quantitativen und qualitativen Analysen bekannten höheren Koordinations- und Betreuungskosten für den Aufbau, die Supervision und die Kontrolle des ausländischen Standorts (Kinkel et al. 2004; Kinkel 2004) am Heimatstandort zu höheren Aufwendungen in den indirekten Gemeinkostenbereichen führen können. Bei markt- und kundenorientierten Auslandsproduzenten, deren Produkte nicht schwergewichtig wieder in den heimischen Produktionsprozess eingeschleust werden müssen und daher weniger koordinationsintensiv sind, scheint sich diese Tendenz zu erhöhten Betreuungs- und Koordinationsaufwendungen dagegen nicht zu zeigen.

\section{INVESTITIONEN IN ANLAGEN SOWIE IN FORSCHUNG UND ENTWICKLUNG}

Wie der Vergleich der Abschreibungsquoten über die verschiedenen Betriebstypen zeigt, entfällt bei Betrieben des Typs Home based player mit im Mittel 7,6 \% ein deutlich höherer Anteil des jährlichen Umsatzes auf Abschreibungen für Maschinen und Anlagen (nicht Gebäude und Grundstücke). Der entsprechende Vergleichswert bei den beiden anderen Verlagerungstypen liegt bei etwa $5 \%$. Das ist ein eindeutiges Indiz dafür, dass gerade Home based player einen höheren Kapitalstock an moderner Produktionstechnik aufweisen als Betriebe, die Verlagerungen ins Ausland vor- genommen haben. Dies deutet darauf hin, dass Letztere bereits in der Vergangenheit weniger in neue Produktionseinrichtungen am deutschen Betriebsstandort investiert haben als Betriebe des Typs Home based player und dies mit hoher Wahrscheinlichkeit auch in Zukunft nicht grundsätzlich ändern werden. Es ist demnach davon auszugehen, dass Investitionen in die Wettbewerbsfähigkeit des deutschen Betriebsstandortes zumindest teilweise durch Investitionen in neue bzw. bereits bestehende ausländische Produktionsstandorte substituiert werden.

Bei der Intensität von Forschung und Entwicklung (FuE), gemessen am Anteil der FuE-Ausgaben am Umsatz, zeigt sich ein deutlicher Rückstand bei Betrieben des Typs 2, die Produktionsverlagerungen aus Kostengründen ins Ausland vornehmen. Mit im Mittel nur etwas mehr als $2 \%$ investieren diese Betriebe signifikant weniger in ihre Zukunftssicherung über Forschung und Entwicklung und damit in mittelfristig innovative Produkte als Home based player bzw. markt- und kundenorientierte Auslandsproduzenten mit einer mittleren FuE-Quote von 5 bzw. 7 \%.

\section{UMSATZRENDITE}

Schließlich ist es interessant, übergreifende Erfolgsindikatoren, wie beispielsweise die Umsatzrendite vor Steuern, für die drei Betriebstypen zu vergleichen. Demnach erwirtschaften Betriebe des Typs Home based player mit im Mittel etwa $6 \%$ die vergleichsweise höchsten Renditen, gefolgt von den markt- und kundenorientierten Auslandsproduzenten mit etwa $5 \%$ und den kostenorientierten Auslandsproduzenten mit unter $4 \%$. Diese Differenzen sollten aufgrund der fehlenden statistischen Signifikanz nicht als Vorsprünge oder Rückstände interpretiert werden. Dennoch zeigt sich, dass Betriebe des Typs Home base player auch bei diesen harten betriebswirtschaftlichen Indikatoren zumindest nicht schwächer als die beiden anderen Typen aufgestellt sind. Auf der anderen Seite sind gerade kostenorientierte Auslandsproduzenten, die mit ihrer Verlagerungsstrategie auf die kurzfristige Verbesserung solcher finanzwirtschaftlicher Indikatoren setzen, hier im Mittel sicherlich nicht überdurchschnittlich erfolgreich. Vor diesem Hintergrund sind kurzfristige finanzielle Erfolgsprognosen, die rein kostengetriebenen Produktionsverlagerungen zugrunde 
liegen, jeweils fallspezifisch sehr gründlich daraufhin zu überprüfen, ob die getroffenen Annahmen realistisch sind.

\subsection{QUALITATIVE BEFUNDE}

Ergänzend zu den quantitativen Analysen wurden vertiefende Experteninterviews mit Mitgliedern der Geschäftsführung und des Betriebsrats bei insgesamt zwölf Automobilzulieferfirmen (je vier Fälle pro Betriebstyp) zu den Entscheidungsmustern und Erfahrungen mit Produktionsstandorten geführt. Übersicht 1 zeigt, wie sich die jeweils vier Betriebe der drei Typen hinsichtlich Betriebsgröße, hergestellter Produkte sowie ihrer Hauptkundenstruktur einordnen.

Demnach sind die vier ausgewählten Fallbeispiele des Typs Home based player (HBP) im Vergleich zu den ausgewählten Fallbeispielen der anderen beiden Betriebstypen eher kleiner und beliefern in keinem der Fälle Volumenhersteller. Kostenorientierte Auslandsproduzenten (KAP) sind zumeist größer, und bei zwei der vier Fälle sind Volumenhersteller als Kundengruppe mit von entscheidender Bedeutung. Die ausgewählten markt- und kundenorientierten Auslandsproduzenten (MAP) weisen die vergleichsweise größten Mitarbeiterzahlen auf und sind in allen Fällen $\mathrm{Au}$ tomobilherstellern aus dem Volumensegment mit Produktionsstätten ins Ausland gefolgt. Diese Strukturunterschiede gilt es bei den folgenden vergleichenden Analysen und Interpretationen zu berücksichtigen.

Aus den Fallstudien lassen sich quer zu den drei Betriebstypen eine Reihe von Erkenntnissen und Erfahrungen ableiten, die wichtige Hinweise auf erfolgreiche Muster internationaler Produktionsstrategien von Automobilzulieferern geben. Die Kernergebnisse sind in der folgenden Übersicht 2 als Synopse zusammengefasst.

\section{UNTERNEHMENSSTRATEGIE UND AUSRICHTUNG IM MARKT}

Erfolgreiche Unternehmen, die gute Renditen erwirtschaften und insgesamt wie auch an ihren deutschen Betriebsstandorten kontinuierlich wachsen, positionieren sich vorrangig über innovative Technologien und Lösungen im Wettbewerb und investieren überdurchschnittlich in Forschung und Entwicklung (FuE). Bei den erfolgreichen Unternehmen finden sich über

Übersicht 1: Einordnung der Automobilzulieferer-Fallbeispiele - nach Betriebstyp, Mitarbeiterzahl, Produkteigenschaften und Hauptkunden -

\begin{tabular}{|c|c|c|c|c|}
\hline & $<499$ & $500-999$ & $1000-4999$ & $>=5000$ \\
\hline $\begin{array}{l}\text { einfache Gummi-/ } \\
\text { Kunststoffprodukte }\end{array}$ & & $\boldsymbol{\Delta} \mathrm{HBP} 2$ & & \\
\hline einfache Metallprodukte & $\Delta \mathrm{HBP} 1$ & & & \\
\hline einfache Elektroprodukte & & - MAP1 & & - KAP2 \\
\hline $\begin{array}{l}\text { komplexe Gummi-/ } \\
\text { Kunststoffprodukte }\end{array}$ & -/ KAP4 & & $\mathbf{\Delta} \mathrm{HBP3}$ & \\
\hline komplexe Metallsysteme & & $\Delta \mathrm{HBP} 4$ & & $\begin{array}{l}\text { MAP } 2 \\
\text { MAP } 4\end{array}$ \\
\hline komplexe Elektrosysteme & & & & $\Delta \mathrm{KAP}_{1}$ \\
\hline Primäre Lieferung an: & $\begin{array}{l}\text { Premiumh } \\
\text { Volumenhe } \\
\text { Sowohl an } \\
\text { first tier-Zu }\end{array}$ & $\begin{array}{l}\text { ler } \\
\text { ler } \\
\text { nium- } \\
\text { rer (d.h }\end{array}$ & taufs & $\begin{array}{l}\text { steller } \\
\text { ier-Ebene) }\end{array}$ \\
\hline
\end{tabular}

Legende: $\mathrm{HBP}=$ Home based player; $\mathrm{KAP}=$ kostenorientierter Auslandsproduzent; $\mathrm{MAP}=$ markt- und kostenorientierter Auslandsproduzent

Quelle: Zusammenstellung der Autoren.

WSI Hans Böckler

alle Betriebstypen hinweg einige, die mehr als $10 \%$ in FuE investieren, einzelne bis zu $18 \%$. Dabei werden die jeweiligen Forschungs- und Entwicklungsaufgaben von den befragten Automobilzulieferern bei allen drei Typen zumindest großteils, vielfach sogar ausschließlich, an deutschen Standorten durchgeführt. Die Entwicklungstiefe ist dabei durchgehend als hoch bis sehr hoch einzustufen. Der vielfach befürchtete Trend, dass die Forschung und Entwicklung der Produktion ins Ausland zunehmend folgt, ist in den (nicht reprä- sentativen) Fallbeispielen bislang nicht $\mathrm{zu}$ beobachten.

Die räumliche Nähe zur Forschung und Entwicklung ist für schnelle und sichere Anlaufprozesse von innovativen, Know-how-intensiven, technologisch anspruchsvollen oder komplexen Lösungen unerlässlich. Die Lead-Kunden für solche Prozesse sind insbesondere deutsche Premiumhersteller. Infolgedessen fahren nahezu alle befragten Automobilzulieferer Produktionsanläufe für neue Produkte und Baureihen ausschließlich an deutschen

\begin{tabular}{|c|c|c|c|}
\hline \multicolumn{4}{|c|}{ Übersicht 2: Kenngrößen und Muster der 12 Fallbeispiele im Überblick } \\
\hline & Home based player & $\begin{array}{l}\text { Kostenorientierte } \\
\text { Auslandsproduzenten }\end{array}$ & $\begin{array}{l}\text { Markt- und } \\
\text { kundenorientierte } \\
\text { Auslandsproduzenten }\end{array}$ \\
\hline Wettbewerbsstrategie & $\begin{array}{l}\text { Qualitäts- oder } \\
\text { Innovationsführer }\end{array}$ & $\begin{array}{l}\text { Innovations- oder } \\
\text { Flexibilitätsführer (3-mal), } \\
\text { Kostenführer (1-mal) }\end{array}$ & $\begin{array}{l}\text { Innovationsführer, } \\
\text { Fast-Follower oder } \\
\text { Kostenführer }\end{array}$ \\
\hline FuE-Quote & $\begin{array}{l}<5 \%(2-\mathrm{mal}) \\
\geq 9 \%(2-\mathrm{mal})\end{array}$ & $4-7 \%$ & $\begin{array}{l}5 \%(1-\mathrm{mal}) \\
\geq 9 \%(3-\mathrm{mal})\end{array}$ \\
\hline Kapitalkostenanteil & k. A. & $5-10 \%$ & $10-15 \%$ \\
\hline Wertschöpfungstiefe & $\begin{array}{l}10 \%(1-\mathrm{mal}) \\
65 \%(3-\mathrm{mal})\end{array}$ & $30-45 \%$ & $\begin{array}{l}20 \%(1-\mathrm{mal}) \\
45 \text { bis über } 60 \% \text { (3-mal) }\end{array}$ \\
\hline Personalkostenanteil & $\begin{array}{l}25-30 \%(3-\mathrm{mal}) \\
>60 \%(1-\mathrm{mal})\end{array}$ & $20-30 \%$ & $\begin{array}{l}15 \%(1-\mathrm{mal}) \\
35 \%(3-\mathrm{mal})\end{array}$ \\
\hline \multirow[t]{2}{*}{ Materialkostenanteil } & $50-65 \%(3-\mathrm{mal})$ & $50-60 \%$ & $45-55 \%(3-\mathrm{mal})$ \\
\hline & $30 \%(1-\mathrm{mal})$ & & $70 \%(1-\mathrm{mal})$ \\
\hline $\begin{array}{l}\text { Anzahl der } \\
\text { Auslandsstandorte }\end{array}$ & $0-2$ & $1-21$ & $3-33$ \\
\hline $\begin{array}{l}\text { Lokal bezogener } \\
\text { Vorleistungsanteil am } \\
\text { Auslandsproduktions- } \\
\text { standort }\end{array}$ & \multicolumn{3}{|c|}{ Größtenteils nur 10 bis $20 \%$ Vorleistungsanteile von lokalen Lieferanten } \\
\hline Quelle: Zusammenstellung & & & WSI $\begin{array}{c}\text { Hans Böckler } \\
\text { Stiftung }\end{array}$ \\
\hline
\end{tabular}


Standorten. Um auf die gestiegenen Anforderungen reagieren zu können, die aus unterschiedlichsten Entwicklungen, wie erweiterten Leistungsumfängen in Entwicklung und Produktion, komplexeren Produktdesigns, verkürzten Entwicklungszeiten oder steileren Anlaufkurven, resultieren, scheint eine enge interne Verzahnung zwischen Entwicklung und Produktion kaum ersetzbar zu sein.

Weiterhin sind erfolgreiche Unternehmen nicht nur bei Produktinnovationen, sondern auch bei Prozessinnovationen führend. Sie investieren überdurchschnittlich - teilweise regelmäßig über $10 \%$ ihres Umsatzes - auch an ihren deutschen Standorten in innovative Herstellverfahren und die kontinuierliche Verbesserung ihrer Organisationsprinzipien, zumeist in Form sogenannter "ganzheitlicher Produktionssysteme“. Hier zeigt sich ein sehr befruchtendes Zusammenspiel zwischen Produktund Prozessinnovationen. Produktinnovative Firmen fahren in nicht allzu langen Abständen immer wieder neue Produktionsprozesse für die neuen Komponenten oder Bauteile hoch, die noch nicht optimal eingeübt sind und bei denen naturgemäß noch beträchtliche Effizienzpotenziale schlummern, die durch technische und organisatorische Prozessinnovationen am deutschen Produktionsstandort abgeschöpft werden können. Auf diese Weise können diese Firmen immer wieder Produktivitätssprünge von mehr als $15 \%$ auch an deutschen Standorten realisieren.

Erfolgsbeispiele können zudem sowohl sehr geringe Fertigungstiefen unter $15 \%$ wie auch überdurchschnittlich hohe Fertigungstiefen von teilweise über $65 \%$ aufweisen. Hier scheint es kein eindeutiges und simples Erfolgsmuster zu geben, auch nicht den oft propagierten Weg des konsequenten Outsourcings zur Reduktion der eigenen Wertschöpfungstiefe. Auch der Personalkostenanteil kann bei erfolgreichen Unternehmen mit 10-15\% bereits weit reduziert sein (vor allem bei kostenorientierten Auslandsproduzenten) oder weiterhin mit Quoten von teilweise über $60 \%$ sehr hoch ausfallen (vor allem bei Home based playern). Auch hier greift eine unreflektierte Vereinfachung geringer Personalkostenanteile als Erfolgsformel zu kurz. Manche Unternehmen verstehen ihren hohen Anteil personalintensiver Tätigkeiten bewusst als Wettbewerbsvorteil und pflegen diesen entsprechend, insbesondere wenn erfahrungs- und wissens- intensive Produkte oder Produktionsprozesse ihre Wertschöpfung prägen.

Kundenbezogene ausländische Produktions- und Montagewerke wurden in der Vergangenheit mehrheitlich auf Initiative deutscher und amerikanischer Volumenhersteller errichtet (following customer). Gerade diese fordern heute auch zunehmend von Automobilzulieferern des Typs markt- und kundenorientierte Auslandsproduzenten, die bislang noch nicht in starkem Maße ausländische Produktionsstätten in Niedriglohnländern aufgebaut haben, dies verstärkt nachzuholen, um selbst local content-Quoten in diesen Ländern erfüllen zu können. Diese von der zentralen Unternehmensplanung der Automobilhersteller (OEM, Original Equipment Manufacturer) vorgegebenen low cost-Quoten gibt der Einkauf nicht selten ungefiltert und mit Nachdruck an die Zulieferer weiter. In der Konsequenz sehen sich manche Automobilzulieferer zum Aufbau oder zur Intensivierung der Produktion in Niedriglohnländern gedrängt, obwohl sie teilweise auch von ihren inländischen Produktionswerken aus die geforderten Preisvorgaben einhalten könnten. Die auf diese Weise „erzwungenen“ Entscheidungen zur Auslandsproduktion vor allem in Osteuropa sind weder betriebswirtschaftlich noch volkswirtschaftlich sinnvoll und werden in der Branche bereits mit Sorge zur Kenntnis genommen (Sihn 2005).

\section{VORGEHEN UND ERFAHRUNGEN BEI STANDORTENTSCHEIDUNGEN}

Neben der Identifikation besonders erfolgversprechender und wachstumsträchtiger Strategiemuster dienten die qualitativen Analysen auch dazu, herauszufinden, welche Defizite die Prozesse der Standortentscheidungsfindung in den betrachteten Unternehmen aufweisen und welche kritischen Faktoren häufig zu wenig beachtet oder falsch eingeschätzt werden. Wie sich zeigte, griffen nur wenige der befragten $\mathrm{Au}$ tomobilzulieferer bei Standortentscheidungen auf ein breites Spektrum oder gar ein standardisiertes Vorgehen und Set von Bewertungsmethoden zurück. Es überwiegen sehr pragmatische, teilweise „hemdsärmlige“ Ansätze, mit denen ein Favorit anhand relativ weniger Kriterien (z. B. Lohnkostenniveau, Entfernung zu den Kunden, Infrastrukturbedingungen oder politischrechtliche Stabilität) ausgewählt wird. Oftmals ist auch die Anzahl der zur Auswahl stehenden Alternativen stark eingegrenzt oder gar von vornherein auf einen Favoriten eingeschränkt, für den dann eine Amortisationsrechnung, eine Kapitalwertoder eine Gewinn- und Verlustrechnung durchgeführt werden (Meyer 2006). Deutsche Standorte sind in vielen Vergleichsrechnungen bewusst nicht als Alternative einbezogen, da durch interne Vorentscheidungen die Suche bereits auf bestimmte Regionen, im Falle kosten- und effizienzorientierter Kalküle zumeist auf osteuropäische Niedriglohnstandorte, eingeschränkt ist. Dies ist prinzipiell zu kritisieren.

Die folgenden wichtigen Kenngrößen, die aus konkreten Erfahrungswerten der analysierten Automobilzulieferer zusammengetragen wurden, dienen als Grundlage, damit auch andere Unternehmen fundierter als bisher einschätzen können, ob eine Standortbewertungsrechnung auf belastbaren Annahmen beruht:

(1) Die kritische Mindestmasse an Produktionsvolumen, die für den Aufbau und mittelfristig tragfähigen Betrieb einer neuen ausländischen Produktionsstätte als notwendig erachtet wird, liegt nach Einschätzung der befragten Automobilzulieferer bei allermindestens 3 bis 5 Mio. $€$ pro Jahr. In diesem Kontext gilt es auch, die Verlässlichkeit der vonseiten der Automobilhersteller zugesagten bzw. prognostizierten Abnahmemengen richtig einzuschätzen. Bei manchen Kunden (insbesondere aus dem Volumenbereich) sind Abschläge auf die Abnahmestückzahlen in der Größenordnung von 10 bis $15 \%$ nicht ungewöhnlich und sollten dementsprechend einkalkuliert werden (vgl. auch KPMG 2005).

(2) Die Anlaufzeiten zur Sicherung der notwendigen Prozessqualität und Produktivität werden nicht selten deutlich unterschätzt. In einem Beispiel betrug ihre Dauer fünfmal länger als ursprünglich geplant, im Mittel war sie etwa doppelt so lang. Die absolute Länge der Anlaufzeiten reicht von sechs Monaten bis zu fünf Jahren, in den meisten Fällen von einem Jahr bis zweieinhalb Jahren. Insgesamt führen lange Anlaufzeiten nicht nur zu hohen Betreuungskosten (s.u.), sondern können auch den geplanten start of production (SOP) gefährden und die errechnete Amortisationsdauer, die für viele Unternehmen das entscheidende Kriterium für oder gegen eine Auslandsinvestition ist (Meyer 2006), empfindlich nach hinten verschieben. 
(3) Die Kosten für die Betreuung und Koordination ausländischer Produktionsstandorte, die von inländischen Standorten, insbesondere dem Stammsitz, erbracht werden, werden oftmals deutlich unterschätzt. In der Anlaufphase einer ausländischen Produktionsstätte können die Betreuungskosten durchaus $10 \%$ der Gesamtkosten ausmachen. Doch auch im eingeschwungenen Zustand können sie noch etwa 2 bis 3,5\% des Umsatzes des ausländischen Produktionsstandorts betragen. Damit fallen teilweise bis zur Hälfte der Personalkosten vor Ort zusätzlich für die laufende Koordination des Standorts an. Für das Benchmarking der Standorte untereinander sowie die spätere Vergabe von Aufträgen im internen Standortwettbewerb ist es äußerst wichtig, die (Inlands-)Standorte, die diese Overhead-Leistungen erbringen, davon rechnerisch zu entlasten.

(4) Der Aufwand für die Qualifizierung der Arbeits- und Führungskräfte des Auslandsstandorts ist nicht zu unterschätzen. Teilweise werden ganze Führungsmannschaften bis zu einem halben Jahr geschult, im Falle von strategischen Werken vorzugsweise am deutschen Stammsitz, bei reinen Just-In-Time- bzw. Just-In-Sequence-Montagewerken vorzugsweise über Paten vor Ort. Die Spanne der Qualifizierungskosten reicht bei unseren Untersuchungsbetrieben, die hierzu verlässliche Angaben machen konnten, von etwa 15 bis etwa $27 \%$ der Personalkosten am ausländischen Standort, was etwa 4,5 bis $9,5 \%$ der Gesamtkosten entspricht.

(5) Erstaunlicherweise geben die befragten Automobilzulieferer an, dass die Materialund Energiekosten im Ausland oftmals höher sind als in Deutschland. Dies betrifft insbesondere spezielle Vorleistungen bestimmter Qualität wie manche Kunststoffoder Aluminiumsorten, die weltweit nur von wenigen Lieferanten bezogen werden können. Die Energiepreise sind in Deutschland in den letzten Jahren im Mittel zwar sehr stark gestiegen, jedoch ist die Industrieenergie in Deutschland günstiger als private Energie.

Produktivitätsvergleiche zwischen deutschen und ausländischen Werken sind grundsätzlich problematisch. Viel entscheidender ist jedoch, dass auch an den bestehenden deutschen Standorten immer wieder relevante Produktivitätspotenziale von
15 bis $30 \%$ geborgen werden können. Diese werden aber insbesondere in kostenorientierten Standortvergleichsrechnungen nur selten berücksichtigt. Vielfach wird auch generell davon ausgegangen, dass die positiven Wirkungen von Modernisierungs- und Reorganisationsmaßnahmen erst mit einer längeren Zeitverzögerung eintreten, während die Vorteile einer kostengünstigen Auslandsproduktion über die geringeren Faktorkosten „quasi sofort“ abgeschöpft werden könnten. Die Erfahrungen der befragten Automobilzulieferer zu realistischen Planungs- und Anlaufzeiten deuten aber darauf hin, dass auch bei der Erlöswirkung von Auslandsproduktionen teilweise mit einem erheblichen Zeitverzug zu rechnen ist. In der Folge ist auch die kumulierte Höhe der realisierbaren Einsparpotenziale durch Auslandsproduktion oftmals limitiert. Einer aktuellen Studie zufolge realisieren die meisten Unternehmen mit ihren neuen Produktionsstätten im Ausland weniger als $10 \%$ Produktionskosteneinsparungen im Vergleich zum inländischen Stammwerk (Abele et al. 2006, S. 34), die zudem noch durch erhöhte Einmal-, Logistik- und Transaktionskosten kompensiert werden können.

Die Erkenntnisse der Fallstudien zeigen schließlich auch, dass sich das Ziel einer low cost sourcing-Strategie, das mit dem Aufbau kostenorientierter Produktionsstätten in Niedriglohnländern manchmal zusätzlich verfolgt wird, nur schwer realisieren lässt. Selbst international erfahrene Unternehmen vertrauen meist auf ihre etablierten Zuliefererstrukturen und beziehen oftmals noch 80 bis $90 \%$ der Vorleistungen nicht lokal vor Ort, sondern lassen sie aus anderen Ländern anliefern. Zudem muss nach den Erfahrungen der befragten Automobilzulieferer für den Bezug von Material im Ausland üblicherweise ein höherer Preis kalkuliert werden als in Deutschland.

\section{Methoden für eine fun- dierte Standortbewer- tungsrechnung}

Die Erfahrungen der befragten Automobilzulieferer legen es in Übereinstimmung mit dem Stand der Literatur zu Standortentscheidungsverfahren nahe (z. B. Kinkel 2003; Hummel 1997; Perlitz 2000; Abele et al. 2006), einen mehrstufigen und auf einem Mix verschiedener Bewertungsmethoden basierenden Ansatz für strategisch fundierte und zukunftsorientierte Standortvergleichsrechnungen zu wählen. Es ist sinnvoll, dass die Unternehmen auf betriebswirtschaftliche Methoden zurückgreifen können, die ihnen nicht völlig fremd sind, die mit überschaubarem Aufwand anwendbar sind und die für verschiedene Bereiche und Beteiligte im Unternehmen, insbesondere auch für die Vertreter der Mitbestimmung, ${ }^{4}$ nachvollziehbar sind. Daher sollte ein mehrstufiger Ansatz folgende drei Teilschritte umfassen:

(1) Ein Kostenstrukturvergleich auf Vollkostenbasis bietet die Möglichkeit, rasch und anschaulich die zukünftig erwarteten Kostenstrukturen der Standortalternativen im eingeschwungenen Zustand zu erfassen. Er vermittelt außerdem, wie sich einerseits die Berücksichtigung der oftmals unterschätzten Koordinationskosten auf den ausländischen Vergleichsstandort auswirkt und wie sich andererseits realisierbare Verbesserungspotenziale in der Kostenstruktur bestehender Standorte niederschlagen. Methodisch sollte hier eine Vollkostenrechnung für die nächsten fünf bis zehn Jahre zur Anwendung kommen, die eine Einschätzung der mittelfristig realisierbaren Kostenstrukturen am jeweiligen Standort erlaubt (Abele et al. 2006 sprechen hier von „Total Landed Costs").

(2) Eine Investitionsrechnung auf Kapitalwertbasis bietet die Möglichkeit, die Zahlungsströme im Zeitverlauf der zugrunde gelegten Vergleichsperiode, die zumindest fünf bis acht Jahre betragen sollte, zu analysieren und belastbare Abschätzungen aufzustellen, ob der Kapitalwert der Investition insgesamt positiv ist und nach welcher Zeitdauer sie sich amortisieren wird (z. B. Abele et al. 2006; Kinkel 2004; Perlitz 2000). Die Berücksichtigung von Anlaufzeiten und -kosten der Produktion an neuen Standorten und der damit verbundenen erhöhten Betreuungskosten kann hier

4 Aufgrund der manchmal asymmetrischen Informationslage ist es für die Betriebsräte in den Unternehmen besonders wichtig, Werkzeuge an die Hand zu bekommen, um vorliegende Standortvergleichsrechnungen systematisch nachvollziehen zu können und hinsichtlich Vollständigkeit der Kriterien sowie Defiziten der verwendeten Methoden einschätzen zu können. 


\section{Abb. 1: Nutzwert-Kapitalwert-Portfolio verschiedener Standort- szenarien für Deutschland und Tschechien}

- fiktives Rechenbeispiel mit realistischen Modelldaten -

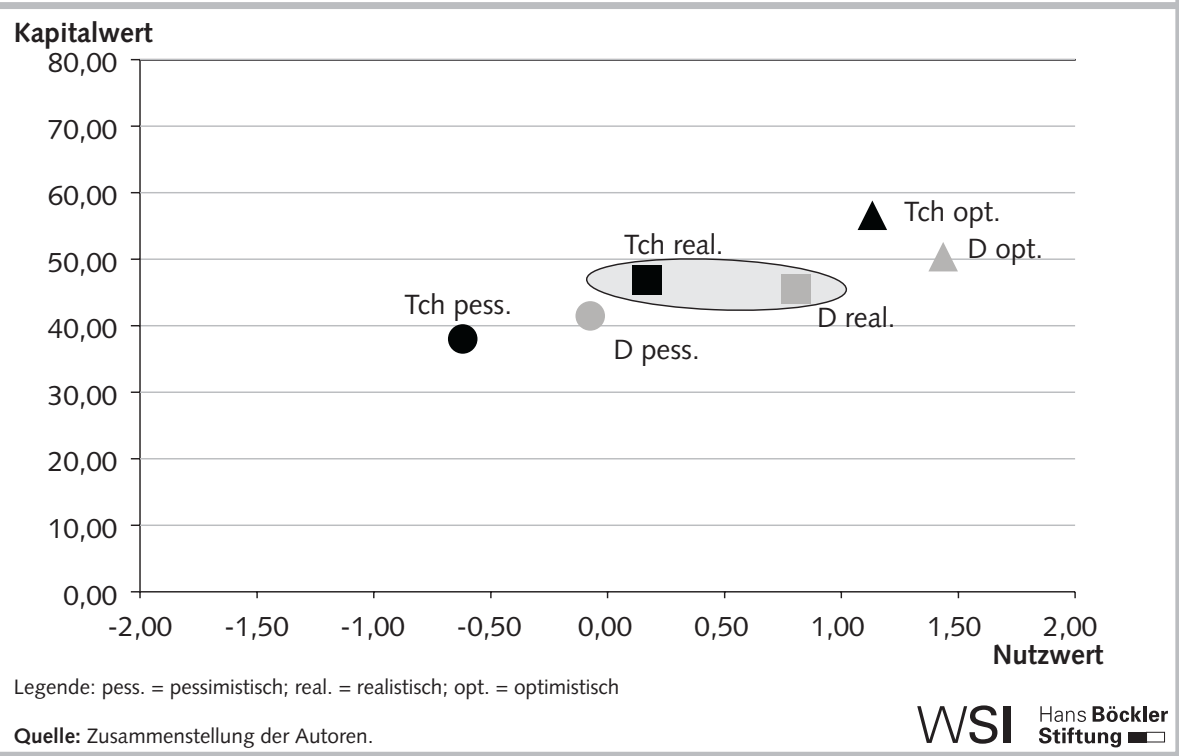

Amortisationszeitpunkte merklich verschieben und sollte daher in jedem Fall mit in die vergleichende Betrachtung einbezogen werden.

(3) Eine Nutzwertanalyse (Zangemeister 1976) der erfolgskritischen qualitativen Standortfaktoren ermöglicht es, nicht monetär quantifizierbare Kriterien, die dennoch von zentraler Bedeutung für den Standorterfolg sein können, angemessen zu berücksichtigen. Beispiele für solche zentralen qualitativen Faktoren sind unter anderem die räumliche und kulturelle Nähe zu Kunden und Absatzmärkten, zu Know-how-Zentren oder innovativen Branchenclustern, zu Lieferanten- und Dienstleisternetzwerken, das Risiko von Know-how-Verlust, die kulturelle Distanz im Werksverbund etc. Zudem wurden auf Basis von verschiedenen Szenarien mögliche zukünftige Gewichtungsveränderungen von Standortfaktoren ermittelt, die an dieser Stelle dynamisch mit berücksichtigt werden können.

Es wird aufgrund der erheblichen Unsicherheiten, die mit einer zukunftsorientierten Standortbewertung für einen Zeitraum von fünf bis zehn Jahren verbunden sind, empfohlen, jeweils drei verschiedene Szenarien - ein optimistisches, realistisches und pessimistisches - je Standortalternative durchzurechnen. Auf diese Weise können Entscheidungen auf Basis von Bandbreiten getroffen werden, die die immanenten Unsicherheiten der Einschätzung zumindest teilweise mit abbilden. Die monetäre und die qualitative Betrachtungsdimension können anschaulich integriert werden, indem den jeweils drei Gesamtnutzenwerten je Standortalternative die jeweiligen Kapitalwerte auf der anderen Achse eines zweidimensionalen NutzwertKapitalwert-Portfolios (Perlitz 2000) gegenübergestellt werden. Ein visuelles Beiternehmens ist mit realistischen Modellannahmen in Abbildung 1 dargestellt.

An diesem Beispiel kann veranschaulicht werden, wie eine Entscheidungsfindung im Abgleich von monetärer (Kapitalwert) und qualitativer (Nutzwert) Dimension unterstützt werden kann. So liegt die deutsche Standortalternative im pessimistischen Fall sowohl beim Nutzwert als auch beim Kapitalwert vor dem tschechischen Vergleichsstandort. Risikoaverse Unternehmer sollten demnach eher dem bestehenden deutschen Produktionsstandort den Vorzug geben. In der "realistischen“ Projektion liegt der bestehende deutsche Standort beim Kapitalwert nur knapp hinter der tschechischen Alternative, zeigt sich beim qualitativen Nutzwert aber deutlich überlegen. Hier müsste der Unternehmer entscheiden, ob ihm der relativ knappe Vorsprung in der monetären Dimension tatsächlich das relativ große Risiko bei den weichen Faktoren wert ist. In der optimistischen Annahme ist die tschechische Standortalternative dem bestehenden deutschen Produktionsstandort beim Kapitalwert am spiel eines fiktiven Automobilzulieferun- deutlichsten überlegen und beim qualitativen Nutzwert am wenigsten deutlich im Hintertreffen. Der Unternehmer hat nun zu entscheiden, ob er den monetären oder den qualitativen Vorteilen eine höhere Priorität einräumt.

Fazit

Die Analyse der internationalen Produktionsstrategien von zwölf deutschen Automobilzulieferern hat gezeigt, dass erfolgreiche Unternehmen, die auch an ihren deutschen Betriebsstandorten kontinuierlich wachsen, überdurchschnittlich in Forschung und Entwicklung investieren und gleichzeitig auch bei Prozessinnovationen führend sind. Zudem werden Forschungsund Entwicklungsaufgaben wie auch Produktionsanläufe für neue Produkte zumindest großteils an deutschen Standorten durchgeführt. Dagegen zeigen sich bei der Fertigungstiefe und beim Personalkostenanteil der Unternehmen keine eindeutigen Erfolgsmuster. Sowohl Automobilzulieferer mit sehr geringen als auch mit sehr hohen Fertigungstiefen oder Personalkostenquoten können sich erfolgreich im Wettbewerb behaupten.

Deutlich geworden ist auch, dass kundenbezogene ausländische Produktionsund Montagewerke von Automobilzulieferern in der Vergangenheit mehrheitlich auf Initiative großer Volumenhersteller errichtet wurden (following customer). Problematisch ist in diesem Kontext die aktuelle Tendenz der OEMs, ihre Zulieferer zunehmend zum Aufbau kostenorientierter Produktionswerke in Niedriglohnländern zu drängen, um selbst local content-Quoten in diesen Ländern erfüllen zu können auch wenn die Zulieferer die geforderten Preisvorgaben von ihren bestehenden Standorten aus einhalten könnten. Die auf diese Weise „erzwungenen“ Entscheidungen zur Produktion in Niedriglohnländern sind weder betriebswirtschaftlich noch volkswirtschaftlich sinnvoll.

Eine besondere Bedeutung kommt Produktivitätsvergleichen von Standortalternativen auf einer fairen Basis zu, das heißt unter Berücksichtigung noch realisierbarer Optimierungspotenziale an den bestehenden Standorten sowie der Zeitdauer bis zu ihrer Freisetzung. Hier ist die vielfach geäußerte Annahme, dass sich die 
positiven Wirkungen von Modernisierungsmaßnahmen an bestehenden Standorten erst später einstellen als die Vorteile einer kostengünstigen Auslandsproduktion, kritisch zu hinterfragen. Die Erfahrungen der befragten Automobilzulieferer zu realistischen Anlaufzeiten deuten darauf hin, dass auch bei der Erlöswirkung von Auslandsproduktionen teilweise mit einem erheblichen Zeitverzug zu rechnen ist.

Aufbauend auf diesen Befunden wurde ein Standortbewertungsinstrument aus drei verschiedenen methodischen Ansätzen konzipiert, um Standortvergleichsrechnungen strategisch fundiert und $\mathrm{zu}$ kunftsorientiert anlegen zu können. Aus der Gesamtheit der jeweiligen Bewertungsmethoden und Zukunftsbilder (optimisti- sches, realistisches und pessimistisches Szenario) erhält der Unternehmer bzw. der Betriebsrat ein facettenreiches und über verschiedene Methoden abgesichertes Gesamtbild je Standortalternative. Die Methoden und Datenblätter, die als Basis für die Vergleichsrechnungen verwendet wurden, können als Vorlage für entsprechende Standortvergleichsrechnungen bei mittelständischen Automobilzulieferern, die sich Standortplanungen oftmals sehr ,handgestrickt" nähern, genutzt werden. ${ }^{5}$ Gerade für die Betriebsräte in den Unternehmen, die oftmals kurzfristig mit einer Bewertung konfrontiert werden und nicht immer über die erforderlichen methodischen Kenntnisse verfügen, ist es besonders wichtig, Werkzeuge an die Hand zu bekommen, um vorliegende Standortvergleichsrechnungen systematisch nachvollziehen und hinsichtlich Vollständigkeit und Defiziten der verwendeten Methoden und Kriterien einschätzen zu können. Damit wird insgesamt die Wahrscheinlichkeit erhöht, Standortentscheidungen zu treffen, die tatsächlich zur Verbesserung der Wettbewerbssituation beitragen.

5 Derzeit wird das beschriebene Instrumentarium als intuitiv zu handhabendes Excel-Tool programmiert und aufbereitet. Es kann von interessierten Unternehmen und Betriebsräten in der Praxis genutzt werden. Die ausführlichen Ergebnisse der Studie werden im Frühjahr 2007 als Buch im SpringerVerlag erscheinen.

\section{LITERATUR}

Abele, E./Kluge, J./Näher, U. (Hrsg.) (2006): Handbuch Globale Produktion, München/Wien

Bassen, A./Behnam, M./Gilbert, D. U. (2001): Internationalisierung des Mittelstands. Ergebnisse einer empirischen Studie zum Internationalisierungsverhalten deutscher mittelständischer Unternehmen, in: Zeitschrift für Betriebswirtschaft 4, S. 413-432

Deutscher Industrie- und Handelskammertag (DIHK) (Hrsg.) (2003): Produktionsverlagerung als Element der Globalisierungsstrategie von Unternehmen. Ergebnisse einer Unternehmensbefragung, Berlin Dreher, C. (2004): Der Manufacturing Technology Action Plan und die Deutsche Produktionsforschung im Europäischen Forschungsraum. Positionspapier der Arbeitsgruppe PROD*EU, Karlsruhe

Hummel, B. (1997): Internationale Standortentscheidung, Freiburg Deutsche Industriebank/Kreditanstalt für Wiederaufbau (IKB/KfW) (Hrsg.) (2004): Studie zu den Auslandsaktivitäten deutscher Unternehmen: Beschäftigungseffekte und Folgen für den Standort Deutschland, Frankfurt/Main

Kinkel, S. (2003): Dynamische Standortbewertung und strategisches Standortcontrolling, Frankfurt/Main

Kinkel, S. (Hrsg.) (2004): Erfolgsfaktor Standortplanung. In- und ausländische Standorte richtig bewerten, Berlin

Kinkel, S./Lay, G./Maloca, S. (2004): Produktionsverlagerungen ins Ausland und Rückverlagerungen, in: Fraunhofer-Institut für System- und Innovationsforschung (Hrsg.): Bericht zum BMF-Forschungsauftrag 8, Karlsruhe
Kinkel, S./Zanker, C. (2006): Internationale Produktions- und Standortstrategien deutscher Automobilzulieferer - Erfolgsmuster und neue Ansätze für fundierte und zukunftsorientierte Standortentscheidungen. Endbericht zum Projekt der Hans-Böckler-Stiftung 2004-644-1, als Publikation im Erscheinen

KPMG (Hrsg.) (2005): Automotive - Globales Standortmanagement in der Automobilzulieferindustrie, Stuttgart

Lay, G./Kinkel, S./Maloca, S. (2003): Stand und Entwicklung der Auslandsproduktion bei Zulieferbetrieben der Automobilindustrie im Vergleich zu anderen Branchen, in: Fraunhofer-Institut für Systemtechnik und Innovationsforschung (Hrsg.): Endbericht zum Projekt-Nr. 2003-522-1 der Hans-Böckler-Stiftung, Karlsruhe

Meyer, T. (2006): Investitionen in Auslandsstandorte: Bewertung und Auswahl, in: Abele, E./Kluge, J./Näher, U. (Hrsg.): Handbuch Globale Produktion, München/Wien, S. 102-143

PA Consulting Group (Hrsg.) (2004): Königsweg ins Ausland? Erfolgsfaktoren für Produktionsverlagerungen, Frankfurt

Perlitz, M. (2000): Internationales Management, Stuttgart Sihn, W. (2005): "Da wird oft blind verlangt“" in: Automobil-Produktion, Dezember, S. 22-24

Zangemeister, C. (1976): Nutzwertanalyse in der Systemtechnik. Eine Methode zur multidimensionalen Bewertung und Auswahl von Projektalternativen, München 